\title{
Useful or just another fad? Staff perceptions of Personal Development Planning
}

\section{Jo Powell}

Birmingham City University, UK

\section{Abstract}

The Quality Assurance Agency (QAA, 2001) suggests that all Higher Education Institutions must offer every student the opportunity to consider their own personal development and promoted Personal Development Planning (PDP) as an important part of supporting and engaging students in their own development whilst within higher education and beyond. The term Personal Development Planning means many things to many people and institutions often appear to adopt their own definition of it. This can be complicated further when faculties or departments within an institution each create their own meaning. Research indicates that staff perceptions of the material they are teaching has a direct link with students' opinions of these activities, so in order to promote and resource PDP effectively it is essential to consider what staff actually think of PDP.

This paper considers the difference between the perceived usefulness of PDP and its elements (reflection, self awareness, action planning and employability), and what it is that impacts upon that view of PDP. The data collected offers the opportunity for both qualitative and quantitative analysis, using free text and ranks of usefulness through an online questionnaire across different faculties within Birmingham City University. The research suggests that both PDP as a process and its elements are perceived as useful by academic staff and it also indicates that the elements are seen as significantly more useful in isolation. It further suggests the rationale for the ranking of PDP as a process was directly linked to perception rather than experience.

Key words: Personal Development Planning; PDP; staff perceptions. 


\section{Introduction}

At Birmingham City University (BCU) an interest in Personal Development Planning (PDP) stemmed from the Progress Files Working Group which was formed as a sub group of the Learning and Teaching Committee in 2001. The focus of that group changed during its existence, and after two years was almost wholly concerned with PDP. Membership of the group gradually changed, leaving a self selected group of dedicated and enthusiastic champions. The view this group held of PDP was holistic, spanning the whole life of a student as opposed to being purely about academic development. The group encouraged and supported faculties to embed or integrate PDP in a way that met theirs and their students' needs. This led to one faculty deciding upon a one size fits all plan of PDP, using a top down approach, with others devolving power to courses and modules to embed where appropriate. The integration of PDP across the organisation has continued to vary from faculty to faculty, with the responsibility for how it is done still decided locally rather than following a fixed university wide strategy. To support staff to understand and integrate both PDP and employability resources were created (Lawton, 2004). The purpose of these was to outline and explain the rationale and importance of both PDP and employability, offering examples and activities for academic staff to embed or use, appropriate for their own courses.

Broadly, within BCU the elements that are considered to be the PDP process are reflection, self awareness, action planning and employability. These elements are both used in isolation or blended as part of modules. How PDP is delivered also varies - in some cases it is peripheral to subject content, in others it is wholly embedded and central to the programme of study. I suspect that the way PDP is delivered leads to a value being associated with it. This varying perception of value of PDP will be explored in this article. The research has focussed on gaining a clearer understanding of perceived usefulness of PDP as a whole in comparison with the constituent elements of the PDP process. This article will not only explore staff perceptions of PDP and its elements, but also the rationale underlying these perceptions. 


\section{Literature review}

The concept of a Progress File was recommended to the sector through the Dearing Report (1997). Dearing (1997, p.3) suggested that Higher Education Institutions (HEls) should develop a progress file that offered students the opportunity to 'monitor, build and reflect upon their personal development'. The progress file was intended to be an active document, developing and changing with the student, offering far more than just a record of achievements. The QAA (2001) stated that students should have the opportunity to consider their own personal development within the Higher Education (HE) environment and therefore the opportunity for students to consider their own personal development should be mandatory and operational across the whole HE system for all awards by 2005/06. Fallows and Steven (2000, p.76) suggested that 'higher education in particular must provide graduates with the skills to be able to operate professionally within the environment required for the 'learning age' or 'learning society'. The importance of recognition and recording of skills had been mooted for some time (Harvey et al., 1997) and PDP was a process which could support this. The definition offered by the QAA (2001, p.2) was 'a structured and supported process undertaken by an individual to reflect upon their own learning, performance and/or achievement and to plan for their personal, educational and career development'.

The Dearing Report and the QAA Guidelines outlined PDP type activities that had been in use in HE for many years (Slight and Bloxham, 2005). In particular the idea of the reflective practitioner (Schön, 1983) had been used widely in vocational subjects and integrated into professional training such as nursing and teaching. As well as the QAA Guidelines, further support for PDP was offered by the Higher Education Academy (HEA, 2005) who outlined characteristics of effective PDP practice in universities. HEA suggested that for PDP to be effective, integration with mainstream academic activities as well as links to learning and programme outcomes was an important aspect. Also support and endorsement by both the academic staff and the institution as a whole was advocated.

Difficulties with integrating PDP into the curriculum have been highlighted by various authors. Davis and Mannion (2005, p.3) suggested from their research that 'PDP was not seen as knowledge development nor was it discipline specific, so, it was not an easy task to convince the academics of its value'. While Slight and Bloxham (2005, p.11) suggest 'current political regimes provide active encouragement for degrees that demonstrate 
relevance to the labour market while critics lament the devaluing of knowledge for knowledge's sake'. What may complicate matters further is the links between PDP and employability (Ward, 2006; 2009).

PDP sits uncomfortably with some academic staff who have concerns for their competence to deliver material outside of their area of expertise (Clegg and Bradley, $2006 \mathrm{~b}$ ), and other fears over the pedagogic value of PDP and it not being seen as part of their role (Clegg and Bradley, 2006b). These views may be shaped by their discipline and organisational culture (Henkel, 2000) and are sometimes entrenched in tradition (Clegg and Bradley, 2006a)

The majority of published studies examine student experiences of PDP (Gough et al., 2003), using the student body as the research sample. This supported the notion that the experience students have of PDP derived from the academic staff that teach them (QAA, 2009). For staff and students to benefit from personal development planning as a concept both the diverse student body and the large numbers of teaching staff need to develop an understanding of both the purpose of PDP and how it can affect their practice and learning (Clegg and Bradley, 2006a).

'The success of a PDP framework depends on the engagement of and the essential value brought to the process by academic staff combined with management support' (QAA Scotland, 2009, p.28). In their report, QAA Scotland further suggested that for student engagement with PDP, staff must be completely aware of, informed and engaged with both the process and the underlying concept and principles of PDP. Without this clarity students are likely to receive conflicting messages about the value and importance of PDP, and thus not give it sufficient attention or value.

Varnava and James (2005) investigated the lack of engagement of tutors and students in the implementation of a paper-based progress file as part of the personal tutoring system at the law school at Glamorgan University. Lack of time and belief in the core values of PDP by tutors, and a lack of understanding by students were the main reasons given for the poor involvement and belief in PDP. Both of these areas are considered to be integral in the embedding of PDP at institutional level by QAA (2009) which outlined key actions for the effective implementation of PDP within HEls. Key actions were 1) ensuring the 'views of stakeholders (such as students, staff and employers)' were sought to 'inform the 
continuous enhancement of PDP Practice' and 2) 'senior management commitment and support for PDP' (QAA, 2009, p.10).

Quinton and Smallbone (2008, P.107) gave consideration to what makes the implementation of PDP successful or not. They suggested that:

...successful implementation requires a blend of the following five areas of good practice: the effective and appropriate use of technology; internal staff champions; support for all staff involved in delivering PDP; clear and meaningful communication with students; and the capture of the institution's cumulative experience over time. Good practice within the conceptual aspects of PDP would include a clear vision of where responsibility lies for PDP within a university, a shared understanding of the purpose of PDP and the promotion of a PDP culture which engages both students and staff.

Ward (2001) discussed the need to get the right balance between overarching institutional policy and getting what he described as psychological engagement with PDP by both staff and students. Therefore the way in which PDP is introduced or embedded within an institution is of utmost importance. When the balance is not gained, a clear understanding and a dedication to PDP cannot be achieved.

\section{Method}

The literature review outlines how important staff are in the successful implementation of PDP. This research considers the perception staff have of the usefulness of PDP and its core elements at BCU (reflection, self awareness, action planning and employability); and any underlying rationale that informed opinions. Ethical approval was sought through the BCU Education Faculty Ethics Committee and a research questionnaire was designed to gather both qualitative and quantitative data from participants to gain an insight into how useful staff perceive PDP to be and what they think it actually is.

The questionnaire was available online in February and March 2009 and open to all 1560 academic staff at BCU from all disciplines. Awareness of the research was raised through 
Learning and Teaching Fellows in each faculty, e-mails to all staff and message board postings on the university intranet to which all staff have access, however it is impossible to state that all staff were aware of the opportunity to participate. The questionnaire took approximately 15 minutes to complete if all questions were answered fully. The questionnaire was externally hosted, which secured participant anonymity, offering staff the opportunity to be honest and open without fear of identification.

Staff were asked to rate their perceptions of how useful they thought PDP was to their students on a Likert scale from 1 (Useless) to 4 (Very Useful). They were further asked to explain why that rating was given. Staff were also asked how useful they thought it was to provide students with the opportunity to: reflect on their own skills; to become self aware; to plan to develop their skills; and to enhance their employability. This was based purely on a Likert scale rating. The data gained from the questionnaire was split into qualitative and quantitative responses and then analysed. The quantitative data was examined using the computer software SPSS to check for any significance, leaving the qualitative free text data to be explored and considered to see if any themes emerged.

\section{Results and discussion}

125 responses were received from academic and learning support staff from across the institution. From the data two main areas were investigated - a statistical analysis of the difference in perceived usefulness of PDP and its elements and a qualitative consideration of the reasons for PDP being ranked as it was.

The responses from the questionnaire fell into four categories outlining staff perceptions of usefulness from useless to very useful. Figure 1 outlines the number of ratings of how PDP and each of the elements were ranked.

Figure 1.

\begin{tabular}{|l|l|l|l|l|l|}
\hline & PDP & Reflection & $\begin{array}{l}\text { Self } \\
\text { Awareness }\end{array}$ & $\begin{array}{l}\text { Action } \\
\text { Planning }\end{array}$ & Employability \\
\hline Useless & $2.8 \%$ & $1.9 \%$ & $2.8 \%$ & $3.7 \%$ & $2.8 \%$ \\
\hline Not Very Useful & $15.0 \%$ & $4.6 \%$ & $4.6 \%$ & $5.5 \%$ & $4.6 \%$ \\
\hline Useful & $38.3 \%$ & $29.6 \%$ & $26.6 \%$ & $33.0 \%$ & $28.7 \%$ \\
\hline Very Useful & $43.9 \%$ & $63.9 \%$ & $66.1 \%$ & $57.8 \%$ & $63.9 \%$ \\
\hline
\end{tabular}


Figure 1 shows that both PDP and its elements are seen as useful; with only $17.8 \%$ of participants considering PDP to be useless or not very useful. When ranking the elements, the percentage of participants who perceived them to be useless or not very useful drops to: $6.5 \%$ for reflection, $7.4 \%$ for self awareness, $9.2 \%$ for action planning and $7.4 \%$ for employability. The data indicated that PDP was seen as less useful than the elements of the PDP process. To consider this further a Wilcoxon signed ranks non-parametric test was carried out on the data set. The results from this indicated that PDP was seen as significantly less useful than its elements $(p \leq 0.001)$. The research data also offered the opportunity to consider if there were any differences in perception using faculty as a variable. When the data was considered looking at the 6 faculties within the institution it demonstrated lower significance levels than the overall data set.

The data was again analysed using Wilcoxon signed ranks with the data split by faculties. In the Birmingham Institute of Art and Design both reflection and action planning were seen as significantly more useful than PDP ( $p \leq 0.05)$; and in the Health faculty both self awareness and action planning were seen as significantly more useful $(p \leq 0.05)$. The results from the other four faculties showed no significant difference in perceived usefulness of PDP and its elements. These differences are intriguing and an interesting subject for further consideration, they are however very difficult to comment on based upon the current data.

From a starting point that PDP as a process is seen as useful, the data was then unpicked and interpreted to understand why its elements were seen as even more useful. One explanation could be due to the understanding of the terminology or concept being muddled or uncertain. Another factor is that PDP is seen by some people as an inconvenient and irrelevant interruption into delivering subject content. This is something that would benefit from further investigation to gain a clearer understanding. The qualitative analysis below however, does go some way to clarifying the underlying rationale for their perception of the PDP process.

The second area considered by the research was the rationale given for how they ranked the usefulness of PDP. In order to complete the analysis the data was explored to see if any themes emerged; these were then pooled together and split into clusters of interlinked themes. From this process the responses fell into two categories; one in which the opinion appeared to be based in or linked to their own or their students' experience; and the 
second grouping that appeared to be unsubstantiated perception. Figure 2 outlines the percentage of responses falling into each category. In both categories it was perception that influenced ratings the most.

Figure 2.

\begin{tabular}{|l|l|l|}
\hline & Perception & Personal Experience \\
\hline $\begin{array}{l}\text { Useless or Not Very } \\
\text { Useful }\end{array}$ & $72.2 \%$ & $27.8 \%$ \\
\hline $\begin{array}{l}\text { Useful or } \\
\text { Very Useful }\end{array}$ & $70.7 \%$ & $29.3 \%$ \\
\hline
\end{tabular}

When PDP was ranked as useless or not very useful, the perception offered appeared to be based around a view that PDP was a concept or fixed structure imposed upon the curriculum by the institution. It was also claimed that the attitude that staff hold is also mirrored by students or vice versa. Examples of reasoning include:

We continually get barraged with new 'concepts'. (Participant 35)

They regard it as an imposition, I agree. (Participant 75)

Students do not grasp the significance of it. However, this may be because most staff do not engage with the process. (Participant 62)

Often comments from this group showed judgements and a resistance to integrate or engage in PDP. The reason given for this is that PDP is perceived as 'an imposition' or often because of the perception that students are not able, or not willing, to engage within the PDP process.

There are also links with the work of Clegg and Bradley (2006b) in relation to the pedagogic value of PDP. Staff commented that they failed to see the value of such work and that it took time away from subject delivery and the quality and commitment to learning and teaching. One participant stated:

Most have little pedagogical value and take time away from the valuable commitment to learning and teaching that many of us have. (Participant 35) 
Looking at the reasons participants gave when they rated PDP as useful or better, their experiences were often based on feedback or experience of students. The comments below indicate that staff often saw or experienced change in student perceptions after some time, and not always immediately:

We've had students come back to us after graduation saying 'now I get it'. (Participant 47)

I sometimes hear students in final year remark how they now appreciate the value of what has been included in this field. (Participant 87)

Because I would like it! (Participant 124)

This indicates not only is the experience of their students important and influential, but also their own personal experience informs staff perceptions of the value of PDP.

The split between experience and perception is a considerable one. This may indicate that for many staff there is a lack of experience of PDP, leaving only the negative opinions that they shared within this data set. This lack of experience may be due to the responsibilities staff have over curriculum design or the perception of what their teaching role is. This gives backing to the findings of the QAA Scotland (2009) in that for PDP to be effective staff must be engaged, and for them to be engaged they must understand and see the purpose of PDP.

When PDP was seen as useful or very useful it is clear that participants have a belief in the process. The belief they hold is that PDP is necessary for the future development of students both within education and beyond. This is indicated by comments such as:

Developing themselves to become useful within their chosen specialism equips them for the real world. (Participant 103)

I think that the experience of compiling the profile will be an empowering and enabling experience for students. (Participant 41) 
There is clearly a link to current literature in relation to the connectedness of staff and student perceptions (Varnava and James, 2005) with one participant suggesting:

It is dependent on how they engage with the concept; which in turn is dependent on how it is put across. (Participant 12)

There were some comments from participants outlining that sufficient resources or support is not available:

Too many students and not enough staff to implement it. (Participant 97)

We use tools inappropriate for the task. (Participant 62)

Some staff have a fixed perception of what PDP is e.g. an externally assessed thing rather than a process that can be used personally to develop oneself:

Students are being asked to internalise the logic of new managerialism by reproducing the assumptions of presenting an apparently reflexive self subject to external scrutiny. (Participant 35)

This suggests that often academic staff do not see PDP as a personal exploration. Instead it is seen as 'a module that has to be passed' (Participant 52) or 'death by reflection' (Participant 62).

\section{Conclusion}

The aim of this article was to report on and interpret the perceptions of staff at BCU in relation to PDP and its elements. The findings were based on a relatively small sample drawn from one institution and offering a broadly representative sample from each faculty. The respondents generally regarded PDP as positive, this could be as participants were more willing to share positive opinions rather than negative. Due to the methods adopted in recruiting participants those who took part chose to do so. In maintaining their anonymity it has not been possible to draw any conclusions about their reasons for taking 
part. However, is it possible to infer that those that participated genuinely see PDP as a useful concept.

Although PDP was seen as a useful process by many, some staff held a view that PDP is a political inconvenience; an intrusion to the academic culture; and an undesirable addition to their current workload, which reflects other research (Quinton and Smallbone, 2008). Often this same group of academic staff saw it as a fixed procedure rather than a holistic process. These perceptions outlined may have been impacted upon by a lack of understanding or a particular interpretation of what PDP is. This certainly appeared in the data and would be at odds with the overarching holistic interpretation the original Progress Files Working Group at BCU had in relation to PDP.

What is also interesting is that there is a significant difference between how PDP and its elements are regarded by academic staff with each of the elements being seen as significantly more useful than PDP as a whole. My inclination based on my experience and the longstanding use of the elements (Schön, 1983; Slight and Bloxham, 2005) is to believe the elements offer more clarity in what they actually are and how they can be used to support student learning.

It is clear that there are many academic staff within the institution that believe in, and value the PDP process. From these it is anticipated that clear communication is taking place with students about the purpose and value of PDP. Further investigation into this area may support the premise that staff communicate that value to students (QAA, 2009).

What the research has demonstrated very clearly is that at BCU over $82 \%$ of the participants saw PDP as either useful or very useful, this number increases even further in relation to each of the elements of PDP. This would indicate that there are a number of staff who speak highly of this process, this bodes well with the suggestions of Quinton and Smallbone (2008) in relation to internal staff champions being required to successfully implement PDP.

The data indicated that there is clear and meaningful communication with students. One possible avenue for future research is the consideration of student perception and experience of PDP at BCU to see if there is a comparison with that of staff. 
This research offers a snap shot of the views of some BCU staff and how they view PDP in one given moment. It does not claim to offer transferability across the sector, rather proposes some interesting data and interpretations that may be worth looking into at different institutions and further and deeper exploration at BCU.

The next stage here at BCU is to start to untangle individual definitions to understand what staff think PDP is. This may offer further clarification on their ratings, and also outline some of the preconceptions and misunderstandings that exist. This will then allow a clear direction institutionally to develop greater knowledge and understanding of what we mean by PDP.

\section{Acknowledgement}

This paper is an outcome of the National Action Research Network on researching and evaluating Personal Development Planning and e-Portfolio practice project (2007-2010). The project was led by the University of Bolton in association with the University of Worcester and Centre for Recording Achievement, and in national collaboration with the University of Bedfordshire, Bournemouth University and University of Bradford. The project was funded by the Higher Education Academy, National Teaching Fellowship Project strand. More details about the project can be found at: http://www.recordingachievement.org/research/narn-tree.html.

\section{References}

Clegg, S. and Bradley, S. (2006a) 'Models of personal development planning: practice and processes', British Educational Research Journal, 32(1), pp. 57-76.

Clegg, S. and Bradley, S. (2006b) 'The implementation of progress files in higher education: reflection as national policy', Higher Education, 51, pp. 465-486. 
Davis, M. and Mannion, M. (2005) 'Integrating personal development planning into the curriculum', Enhancing Student Employability: Higher Education and Workforce Development: 9th Quality in Higher Education International Seminar. Birmingham 27-28 January.

Dearing, R. (1997) Higher Education in the learning society: report of the National Committee of Inquiry in Higher Education. London: Stationery Office.

Fallows, S. and Steven, C. (2000) 'Building employability skills into the higher education curriculum: a university wide initiative', Education and Training, 42(2), pp. 75-82.

Gough, D., Kiwan, D., Sutcliffe, K., Simpson, D. and Houghton, N. (2003) A systematic map and synthesis review of the effectiveness of personal development planning for improving student learning. London: EPPI-Centre, Social Science Research Unit. Available at:

http://eppi.ioe.ac.uk/cms/LinkClick.aspx?fileticket=XSIHVsvXA10\%3d\&tabid=309\&m id=1188\&language=en-US (Accessed: 23 April 2010).

Harvey, L., Moon, S. and Geall, V. (1997) Graduates' work: organisational change and students' attributes. Birmingham: Centre for Research into Quality.

Henkel, M. (2000) Academic identities and policy change in higher education. London: Jessica Kingsley.

Higher Education Academy (HEA) (2005) Guide for busy academics no.1: personal development planning. York: HEA. Available at: http://www.heacademy.ac.uk/resources/detail/resource database/id66 guide for b usy academics no1 (Accessed: 27 May 2010).

Lawton, R. (2004) Embedding PDP and employability. Available at: http://www.bcu.ac.uk/docs/downloads/studentservices/employability/Embedding\%2 OPDP\%20and\%20Employability\%20-\%20Complete\%20file\%202008.pdf (Accessed: 26 Feb 2010). 
Quality Assurance Agency (QAA) (2001) Guidelines for HE progress files. Available at: http://www.qaa.ac.uk/academicinfrastructure/progressFiles/guidelines/progfile2001. asp (Accessed: 11 May 2010).

Quality Assurance Agency (2009) Personal development planning: guidance for institutional policy and practice in higher education. Gloucester: QAA. Available at: http://www.qaa.ac.uk/academicinfrastructure/progressFiles/guidelines/PDP/PDPgui de.pdf (Accessed: 14 April 2010).

Quality Assurance Agency Scotland (2009) A toolkit for enhancing personal development planning strategy, policy and practice in higher education institutions. Available at: http://www.qaa.ac.uk/scotland/PDP/PDP Toolkit Final.pdf (Accessed: 12 May 2010).

Quinton, S. and Smallbone, T. (2008) 'PDP implementation at English universities: what are the issues?', Journal of Further and Higher Education, 32(2), pp. 99-109.

Schön, D. (1983) The reflective practitioner: how professionals think in action. New York: Basic Books.

Slight, A. and Bloxham, S. (2005) 'Embedding personal development planning into the social sciences', Learning and Teaching in the Social Science, 2(3), pp. 191-206.

Varnava, T. and James, H. (2005) 'Reflections on PDP in law', Socio-legal Studies Association Annual Conference. University of Liverpool $30^{\text {th }}$ March $-1^{\text {st }}$ April.

Ward, R. (2001) 'Developing and implementing institutional policy on PDP: setting the scene', in Jackson, N. and Ward, R. (eds.) Personal development planning: institutional case studies. York: Learning and Teaching Support Network, p.2-6.

Ward, R. (2006) Personal development planning and employability: learning and employability series 2. York: The Higher Education Academy.

Ward, R. (2009) Personal development planning and employability (revised edn): learning and employability series 2. York: The Higher Education Academy. 


\section{Author details}

Jo Powell is the Tutor for PDP and Employability at Birmingham City University. Her role is to support and develop Personal Development Planning and Employability within and outside of the curriculum through supporting staff, developing resources and championing good practice. 